\title{
BMJ Open Normative visual acuity in Chinese preschoolers aged 36 to $<48$ months as measured with the linear HOTV chart: the Yuhuatai Pediatric Eye Disease Study
}

Hui Zhu, ${ }^{1}$ Dan Huang, ${ }^{1}$ Qigang Sun, ${ }^{1}$ Hui Ding, ${ }^{2}$ Jing Bai, ${ }^{2}$ Ji Chen, ${ }^{2}$ Xuejuan Chen, ${ }^{1}$ Yue Wang, ${ }^{1}$ Xiaohan Zhang, ${ }^{1}$ Jing Wang, ${ }^{3}$ Xinying $\mathrm{Li}^{3}{ }^{3} \mathrm{Hu} \mathrm{Liu}^{1}$

To cite: Zhu H, Huang D, Sun $Q$, et al. Normative visual acuity in Chinese preschoolers aged 36 to $<48$ months as measured with the linear HOTV chart: the Yuhuatai Pediatric Eye Disease Study. BMJ Open 2017;7:e014866. doi:10.1136/ bmjopen-2016-014866

- Prepublication history for this paper is available online. To view these files please visit the journal online (http://dx.doi org/10.1136/bmjopen-2016014866)

$\mathrm{HZ}$, DH and QS contributed equally.

Received 22 October 2016 Revised 15 March 2017 Accepted 12 May 2017

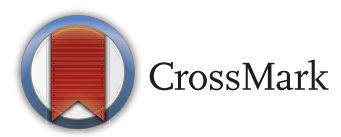

${ }^{1}$ Department of ophthalmology, The First Affiliated Hospital with Nanjing Medical University, Nanjing, China

${ }^{2}$ Maternal and Child Healthcare Hospital of Yuhuatai District,

Nanjing, China

${ }^{3}$ Jinling vision care center for children and adolescents, Nanjing, China

Correspondence to

Dr. Hu Liu; dr_liuhu66@163. com

\section{ABSTRACT}

Objectives To document population-based normative data for uncorrected visual acuity (UCVA) in Chinese preschoolers aged 36 to $<48$ months without any sightaffecting abnormalities and to evaluate its effectiveness for vision referral.

Methods In a population-based cohort of children in the Yuhuatai Pediatric Eye Disease Study, UCVA was measured by using the linear HOTV chart, followed by other ocular examinations. Reference population was defined as children without ophthalmic abnormalities or refractive error. Normative UCVA was obtained from the reference population. The UCVA referral cut-off was defined as the lowest fifth percentile of the normative distribution of UCVA.

Results The analysis cohort consisted of 1606 Chinese preschoolers aged 36 to $<48$ months. Among them, a total of 791 children were included in the reference population. The 5th, 50th and 95th percentiles of the UCVA distribution in the reference population were 20/40, 20/32 and $20 / 25$, respectively. UCVA improved with increasing age $(p<0.0001)$, but worsen if prematurity was presented $(p=0.041)$. Using the fifth percentile, UCVA cut-off from the reference population generated referral rates of $26.9 \%$ in the general population, and detected more than $86 \%$ of amblyopia cases.

Conclusions We propose that UCVA no better than 20/40 measured by linear HOTV chart should be a referral cut-off for Chinese preschoolers aged 36 to $<48$ months. Most amblyopia cases can be identified with this age-specific and chart-specific UCVA cut-off.

\section{INTRODUCTION}

Accurate and timely determination of visual acuity (VA) is the basis of clinical management of many ocular conditions in children. ${ }^{1}$ The American Academy of Pediatrics (AAP) recommended that objective evaluation of VA be initiated by 36 months of age. ${ }^{2}$ Unfortunately, because of the different charts used in different countries and the developing visual and cognitive systems, normative VA

\section{Strengths and limitations of this study}

- This is a population-based study, comprising 1606 preschoolers aged 36 to $<48$ months.

- Normative uncorrected visual acuity was obtained from 791 children without any sight-affecting abnormalities among the population-based cohort.

- The linear HOTV chart, which was recommended when measuring visual acuity in 36-month-old and 48-month-old children, was used in the study.

- Only children with suspected abnormalities underwent cycloplegic refraction, which may impact the detection of sight-affecting refractive errors.

- $11.1 \%$ children were not testable when doing the HOTV test and were excluded from the analysis.

levels in children of preschool age are not uniform internationally. ${ }^{3-5}$ This makes it difficult to accurately set referral criteria for vision screening and to effectively monitor and manage eye conditions, requiring the development of age-specific and chart-specific normative VA.

For ensuring measures accurately reflect what could be expected in healthy eyes, VA norms need to be established on the basis of normative data, obtained by studying reference populations of children who do not have sight-affecting ocular conditions. Normative data of this kind have been reported in the Multi-Ethnic Pediatric Eye Disease Study (MEPEDS), ${ }^{6}$ and the Sydney Paediatric Eye Disease Study (SPEDS). ${ }^{7}$ The samples of preschool children were African American and Hispanic in MEPEDS, and predominantly European Caucasian in SPEDS. Given that ethnicity and socioeconomic status (SES) have potential effects on the level of VA measured, 
population-specific norms still need to be established for Chinese preschoolers. ${ }^{6-9}$

In China, only the Shenzhen Kindergarten Eye Study (SKES) provided population-based normative data for VA in children 36 to 72 months old..$^{10}$ However, in SKES, VA was measured by the Early Treatment Diabetic Retinopathy Study (ETDRS) Tumbling-E chart, which is commonly used in children aged 66 months and older but too difficult for younger children to complete. ${ }^{11}$ The HOTV or Lea Symbols chart tests were recommended by AAP when measuring VA in 36-month-old and 48-month-old children. ${ }^{12}$

This report aims to provide the population-based normative distribution of monocular uncorrected VA (UCVA) and interocular differences (IOD) in UCVA in Chinese children aged 36 to $<48$ months by using the linear HOTV chart, and explore appropriate UCVA criteria for referral of cases of suspected amblyopia and refractive error. This analysis is part of the Yuhuatai Pediatric Eye Disease Study (YPEDS).

\section{METHODS}

\section{Study population}

YPEDS is an ongoing prospective population-based vision screening study, with the specific aims of establishing a systematic database on refraction, VA, ocular biometric parameters, ocular position and other ophthalmic measures, exploring the development role of vision, and estimating the burden of common paediatric ocular disorders of preschool children. ${ }^{13}$ All of the children, who resided in Yuhuatai District, were born between September 2011 and August 2012, and were about to enter a kindergarten in Yuhuatai District, were invited to participate in YPEDS and to undergo a further vision examination in addition to a compulsory health examination. The data presented in this paper were obtained from July 2015 to August 2015, when these children were 36 to $<48$ months old.

This study was approved by the Ethics Committee of Nanjing Medical University and followed the tenets of the Declaration of Helsinki. Written informed consent was obtained from the parents or legal representatives of all the participating children.

\section{Ocular examinations}

A detailed parental interview was conducted, including questions regarding parental education level, monthly family income, employment of parent/s, history of pregnancy and delivery, and parental history of smoking and drinking during pregnancy. Comprehensive eye examinations included distance VA, ocular alignment and motility, non-cycloplegic refractive error measurement, anterior segment examination, fundus evaluation by indirect ophthalmoscopy and ocular biometric parameters.

Monocular distance UCVA measurements were attempted, first in the right eye, and then in the left, at $3 \mathrm{~m}$ by using a retroilluminated (ESV1200 Illuminated
Cabinet, GOOD-LITE, USA) linear HOTV logMAR chart (600017, GOOD-LITE, USA) with matching letter card. VA scores were measured in $0.1 \operatorname{logMAR}$ increments from $20 / 100$ to $20 / 16$. A similar, standardised approach to the Amblyopia Treatment Study (ATS) HOTV VA testing protocol was adapted ${ }^{14}$ :

1. Screening: Patch the eye not being tested. Project a 20/100 optotype. If correct, go down a logMAR level and again show a single optotype. Continue through 20/20 with one letter per level until an incorrect response.

2. Phase 1: Move up two letter sizes from the letter size with the incorrect response in screening up to maximum size of 20/100.

a. Present four new letters (if first three new letters are correct, then the fourth does not need to be tested; as soon as two letters are missed, testing of a level stops).

b. If less than three letters are correct, proceed to the next largest size and so on until 3 of 4 are correct. When 3 of 4 are correct, proceed to 'reinforcement'. If 20/100 is failed, stop testing.

c. If three letters are correct, repeat on next smallest optotype. Continue to move to smaller optotypes as long as first 3 or 3 of 4 are correct. If $20 / 16$ is passed, test is over. When two letters on a level are missed, stop and move to 'reinforcement'.

3. Reinforcement: Move up three levels from the level missed in phase 1 and show three successively smaller single letters. If the patient fails phase 1 at $20 / 63$ or $20 / 80$, show three $20 / 100$ letters but still start phase 2 at the level failed in phase 1 . Whether or not all three are correct in reinforcement, proceed to phase 2.

4. Phase 2: Retest the last level failed in phase 1. Continue the test by the same procedure as described for phase 1 , with the exception that if two letters are missed, testing stops.

5. Recording VA: Acuity is the smallest letter size (level) passed in phase 1 or phase 2 .

Non-cycloplegic refraction was performed with the table-mounted autorefractor (R-F10, Cannon, Tokyo, Japan), photorefraction (PlusoptiX GmbH, Nuremberg, Germany) and retinoscopy. Cycloplegic refraction was performed in any of the following conditions: (1) spherical equivalent refraction (SER) obtained from any of the non-cycloplegic refraction measurements was $\leq-0.50 \mathrm{D}$ in either eye, $\geq+1.25 \mathrm{D}$ in either eye, or showed a difference $\geq 0.75$ D interocularly; (2) cylindrical dioptre obtained from any of the non-cycloplegic refraction measurements was $\geq 0.75 \mathrm{D}$ in either eye; (3) UCVA was $<20 / 40$ (logMAR 0.3) in either eye, or $\geq 2$ line IOD; (4) abnormal results in the examinations of ocular alignment and motility, anterior segment examination or fundus evaluation were found. Two drops of $1.0 \%$ cyclopentolate (Cyclogyl, Alcon, Belgium) were instilled 5 min apart, with a third drop administered 20 min later. Cycloplegia was then evaluated after an additional $15 \mathrm{~min}$. Cycloplegia 
was considered complete if a pupillary light reflex was absent. If a light reflex was still detected, another drop of cyclopentolate was administered, and the light reflex was evaluated again after $15 \mathrm{~min}$. Cycloplegic refraction was performed with the table-mounted autorefractor (R-F10, Cannon, Tokyo, Japan). Subjective refraction was then assessed monocularly according to the cycloplegic refraction values. The best-corrected VA (BCVA) was recorded based on monocular subjective refraction, using the same protocol and VA chart as the UCVA measurements.

\section{Definitions}

The criteria of refractive errors were based on the cycloplegic refraction values: myopia was defined as SER $\leq-0.50$ $\mathrm{D}$ in either eye; hyperopia was defined as SER $\geq+2.00 \mathrm{D}$ in either eye; astigmatism was defined as a cylindrical refractive error $\geq 0.75 \mathrm{D}$; anisometropia was defined as an SER difference $\geq 2.00 \mathrm{D}$ interocularly.

To be comparable with SKES, two definitions of amblyopia were adopted in the present report. Amblyopia risk factors should be identified when diagnosing amblyopia in each of the definitions: (1) the American Academy of Ophthalmology (AAO) amblyopia Preferred Practice Pattern guidelines define unilateral amblyopia in preschoolers aged 36 to $<48$ months as an IOD of greater than or equal to two lines of BCVA, and bilateral amblyopia as BCVA less than $20 / 50$ in either eye ${ }^{15}$; (2) the Chinese Ophthalmological Society (COS) guidelines define amblyopia in preschoolers aged 36 to $<48$ months as an IOD of greater than or equal to two lines of BCVA or BCVA less than $20 / 40$ in either eye. ${ }^{16}$

\section{Inclusion and exclusion criteria}

Only Chinese children aged 36 to $<48$ months who were able to complete monocular UCVA testing in both eyes and without neurological problems were included in the analysis and considered as the general study population. The reference population was defined as the children without sight-affecting ocular conditions, including myopia, hyperopia, astigmatism, anisometropia, amblyopia, strabismus, nystagmus, visual axis occlusion or other anterior segment or fundus abnormalities capable of causing visual impairment.

\section{Statistical analysis}

UCVA outcomes were recorded continuously as mean logMAR UCVA or mean logMAR IOD in UCVA and dichotomously as the proportion of children achieving a particular level of UCVA or IOD. The UCVA for right and left eyes in the reference population were highly correlated (Pearson's correlation $=0.721, \mathrm{p}<0.01$ ); thus, right eye UCVA was used to report the distribution of UCVA. The UCVA cut-off for referral was defined as the lowest fifth percentile of the normative distribution of UCVA. Effectiveness in referral for amblyopia or refractive error using the estimated UCVA cut-off was then calculated. Multiple linear regression was used to assess associations of logMAR UCVA with age, gender and other
Table 1 Distribution of UCVA and IOD in the reference population

\begin{tabular}{lc}
\hline & $\mathbf{n ~ ( \% )}$ \\
\hline LogMAR UCVA (Snellen equivalent) & $1(0.1)$ \\
$0.6(20 / 80)$ & $2(0.3)$ \\
$0.5(20 / 63)$ & $6(0.8)$ \\
$0.3(20 / 40)$ & $106(13.4)^{\star}$ \\
$0.2(20 / 32)$ & $478(60.4)^{\star}$ \\
$0.1(20 / 25)$ & $170(21.5)^{\star}$ \\
$0(20 / 20)$ & $27(3.4)$ \\
$-0.1(20 / 16)$ & $1(0.1)$ \\
UCVA IOD, lines (LogMAR equivalent) & \\
$0(0.0)$ & $595(75.2)$ \\
$1(0.1)$ & $181(22.9)$ \\
$2(0.2)$ & $14(1.8)$ \\
$3(0.3)$ & $1(0.1)$ \\
\hline
\end{tabular}

${ }^{*}$ The 5th, 50th and 95th percentiles fell in the UCVA categories of 20/40, 20/32 and 20/25, respectively.

IOD, interocular difference; UCVA, uncorrected visual acuity.

potential risk factors. All analyses were performed by using SPSS V.22.0 (IBM, China) and a 0.05 significance level.

\section{RESULTS}

\section{Study population}

A total of 2300 preschoolers were enrolled in this study, and 1806 participated in the UCVA test (78.5\% responses rate), among which 1606 (88.9\%) were testable in both eyes and considered as the general population. According to the definition outlined in the methods, 791 (49.3\%) children were classified as the reference population. Boys constituted $53.5 \%$ of the reference population and $52.5 \%$ of the general population. There was no statistically significant sex difference in the mean age of the reference population $(\mathrm{p}=0.277)$ and the general population $(\mathrm{p}=0.607)$.

\section{UCVA outcomes}

Table 1 presents the distributions of UCVA in the reference population. About $60 \%$ (478 out of 791 ) of the measured monocular UCVAs fell in the category of 20/32. The 5th, 50th and 95th percentiles fell in the UCVA categories of 20/40, 20/32 and 20/25, respectively.

Age-specific and gender-specific mean logMAR UCVA levels for the right eye in the reference population are shown in table 2. A multivariate linear regression model including age and gender showed that UCVA improved with increasing age $(\mathrm{p}<0.0001)$ but had no association with gender $(p=0.892)$. Risk factors adjusted for age and adjusted for age and gender for UCVA in the reference population are shown in table 3. Only prematurity was significantly associated with poorer UCVA after 
Table 2 Mean logMAR UCVA and IOD in reference generation by age and by gender

\begin{tabular}{|c|c|c|c|}
\hline & $\mathbf{n}$ & $\begin{array}{l}\text { Mean UCVA } \\
\text { (SD) }\end{array}$ & $\begin{array}{l}\text { Mean absolute } \\
\text { UCVA IOD (SD) }\end{array}$ \\
\hline All & 791 & $0.19(0.07)$ & $0.03(0.05)$ \\
\hline \multicolumn{4}{|c|}{ Age in months } \\
\hline 36 to $<42$ & 438 & $0.19(0.08)$ & $0.03(0.05)$ \\
\hline 42 to $<48$ & 353 & $0.18(0.07)$ & $0.02(0.04)$ \\
\hline \multicolumn{4}{|l|}{ Gender } \\
\hline Male & 423 & $0.19(0.08)$ & $0.03(0.05)$ \\
\hline Female & 368 & $0.19(0.07)$ & $0.02(0.05)$ \\
\hline
\end{tabular}

IOD, interocular difference; UCVA, uncorrected visual acuity.

adjustment for age $(\mathrm{p}=0.040)$ or adjusted for age and gender $(\mathrm{p}=0.041)$.

$75.2 \%$ (595 out of 791) of children in the reference population achieved equal vision in both eyes and only $1.9 \%$ had an IOD of two or more lines $(\geq 0.2 \log$ MAR) (table 1). Table 2 shows mean absolute logMAR UCVA IOD in the reference population by age and by gender. A multivariate linear regression model including age and gender showed that absolute UCVA IOD decreased with increasing age $(\mathrm{p}=0.014)$ but had no association with gender $(p=0.227)$.

The mean non-absolute logMAR UCVA IOD (right eye minus left eye $\log$ MAR VA) in the reference population was 0.0023 , with no significant difference from 0 $(p=0.251)$, indicating no influence of the order in which the eyes were tested on UCVA.

\section{Effectiveness of the UCVA and UCVA IOD referral cut-offs}

Using the fifth percentile UCVA cut-off from the reference population (defined as UCVA $\leq 20 / 40$ ) would generate referral rates of $26.9 \%$ (432 cases) in the general population. Table 4 shows the effectiveness in terms of sensitivity, specificity, positive predictive value and negative predictive value in referral for refractive errors or amblyopia using the current UCVA cut-off. A total of $39.5 \%(301 / 762)$ of cases with any refractive error were detected, with a specificity of $84.5 \%$ (713/844). For amblyopia using the AAO definition, the sensitivity and specificity were $86.2 \%(25 / 29)$ and $74.2 \%$ (1170/1577), respectively, which are similar to the findings when using the COS amblyopia definition $(86.7 \%$ and $74.2 \%)$.

When using the UCVA IOD of two or more lines as the criterion, only $4.3 \%$ (69 cases) of the general population would be referred. According to the AAO definition, this criterion detected $20.7 \%(6 / 29)$ of cases with any amblyopia, and $28.6 \%$ (4/14) of cases with unilateral amblyopia. For amblyopia using the COS definition, 26.7\% (8/30) of cases with amblyopia were detected using such criterion.

\section{DISCUSSION}

In China, SKES was the only population-based investigation of UCVA normative distribution in Chinese preschoolers before our study. ${ }^{10}$ In order to be comparable with SKES and also to ensure that children with sight-affecting ocular conditions were excluded, we used the same stringent definition of the 'reference population' as SKES. However, the lowest fifth percentile in our reference population, falling in the UCVA category of 20/40, was two lines better than this found in the 36 -month-old reference population in SKES (20/63). In addition, the 50th percentile in our reference population was one line better than this in SKES (20/32 vs 20/40). Two reasons might contribute to the differences between our study and SKES. First, we measured the UCVA using the HOTV chart, which is recommended and commonly used in Western countries for young children, ${ }^{6} 712$ while SKES used the ETDRS Tumbling E chart which is cognitively harder for the child to understand and thus affect the threshold acuity that can be achieved by the child. ${ }^{17-19}$ Second, smaller sample size of the 36-month-old children (51 cases) in the reference population of SKES might have a significant influence on the results of the UCVA normative distribution.

We are unable to compare the mean logMAR UCVA in our study with that in SKES as SKES did not calculate this.

Table 3 Risk factors adjusted for age and adjusted for age and gender for uncorrected visual acuity in the reference population

\begin{tabular}{|c|c|c|c|c|}
\hline \multirow[b]{2}{*}{ Associated factors } & \multirow{2}{*}{$\begin{array}{l}\text { Adjusted for } \\
\text { age } \\
\text { p Value }\end{array}$} & \multicolumn{3}{|c|}{ Adjusted for age and gender } \\
\hline & & $\beta$ coefficient & $r^{2}$ & p Value \\
\hline Prematurity $<37$ weeks & 0.040 & 0.082 & 0.032 & 0.041 \\
\hline Low birth weight $<2500 \mathrm{~g}$ & 0.163 & 0.054 & 0.028 & 0.163 \\
\hline Maternal smoking during pregnancy & 0.770 & 0.012 & 0.023 & 0.758 \\
\hline Maternal drinking during pregnancy & 0.264 & -0.042 & 0.024 & 0.270 \\
\hline Parental tertiary education (university or college) & 0.755 & -0.012 & 0.024 & 0.754 \\
\hline Monthly family income & 0.685 & -0.019 & 0.016 & 0.689 \\
\hline Employment of one parent & 0.501 & -0.028 & 0.030 & 0.506 \\
\hline Employment of both parents & 0.791 & 0.011 & 0.029 & 0.798 \\
\hline
\end{tabular}


Table 4 Effectiveness in referral for refractive errors or amblyopia using the lowest fifth percentile UCVA cut-off criteria

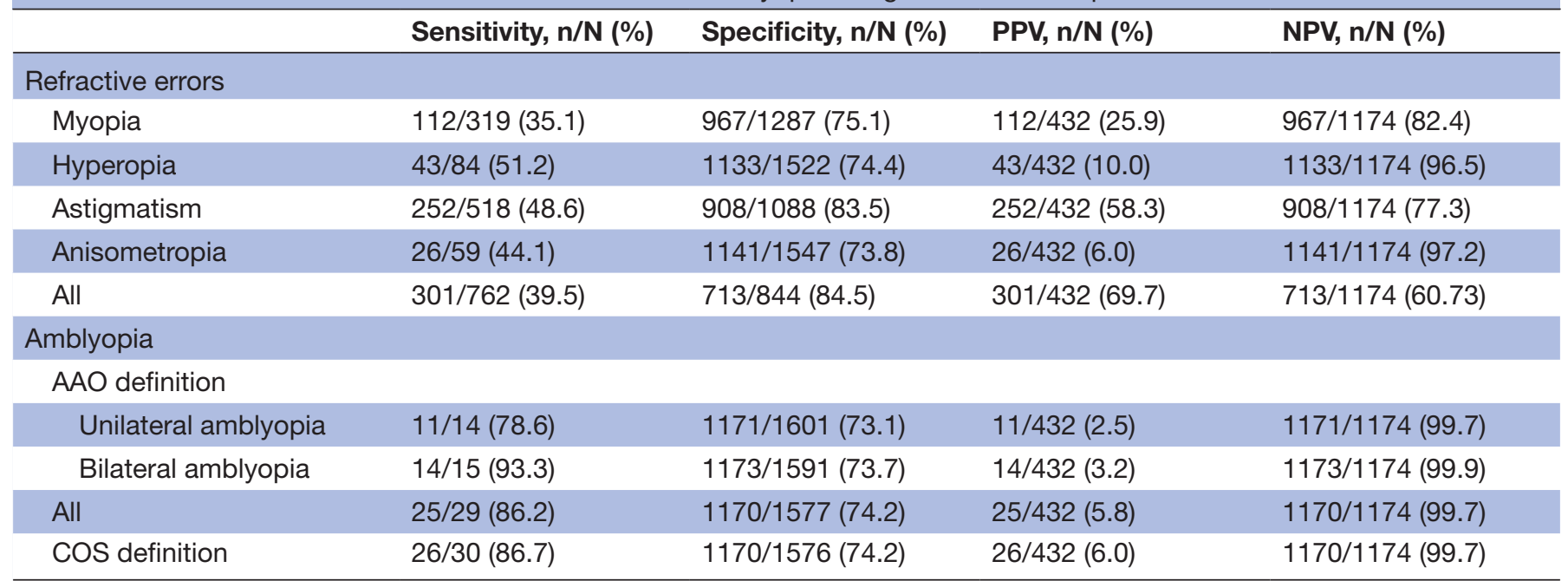

AAO, American Academy of Ophthalmology; COS, Chinese Ophthalmological Society; NPV, negative predictive value; PPV, positive predictive value; UCVA, uncorrected visual acuity.

When our mean logMAR UCVA is compared with this obtained from the African American and Hispanic children without sight-affecting abnormalities in MEPEDS, the mean $\log$ MAR UCVA in our reference population is very close to this established in the MEPEDS 36-month-old to 47 -month-old children using the ATS HOTV (0.19 vs $0.17 \log$ MAR, respectively) ${ }^{6}$ If compared with the results obtained from the European Caucasian and Asian children without sight-affecting abnormalities in SPEDS, the mean logMAR UCVA in our reference population is close to one line worse than this tested with the ATS HOTV in SPEDS ( 0.19 vs $0.09 \operatorname{logMAR}$ for children aged 36 to $<42$ months; 0.18 vs 0.07 for 42 to $<48$ months), and similar to this tested with the linear ETDRS or HOTV logMAR chart ( 0.19 vs $0.22 \operatorname{logMAR}$ for children aged 36 to $<42$ months; 0.18 vs 0.16 for 42 to $<48$ months). ${ }^{7}$ However, the significances of such comparisons are not very clear, because the ATS HOTV VA test, which uses a single letter surrounded by bars optimally placed at half the optotype height from the letter optotype, is quite different from the linear HOTV logMAR chart that we used, and SPEDS did not specify the mean logMAR UCVA when only using linear HOTV $\log$ MAR chart. The better VA by approximately one line (using the ATS HOTV test) found in SPEDS when compared with our study might be due to the following reasons: (1) Non-cycloplegic refraction was performed on part of children in our reference population, which may underdiagnose hyperopia due to accommodative reserves ${ }^{2021}$ and then produce slightly reduced VA in the reference population due to hyperopia; (2) The ATS HOTV is cognitively easier to perform by preschoolers than the linear chart ${ }^{22}$; (3) Leone $e t a l^{7}$ found that East Asian children had significantly poorer VA than their European Caucasian counterparts, 7 which indicated that ethnicity differences might have impacted our findings.
We found improvement in UCVA with increasing age, as has been reported in several studies despite different charts used. ${ }^{671018}{ }^{23}$ However, the difference between UCVA in the 36-month-old to <42-month-old children was only $0.01 \operatorname{logMAR}$ worse than the 42-month-old to <48-month-old children (table 2), which might have no clinical significance. Gender was not associated with UCVA both in our sample and SPEDS, opposite to that reported in MEPEDS. ${ }^{67}$ As for risk factors of UCVA, prematurity was related with worse UCVA in our reference population, which was consistent with the finding in SPEDS. However, the $\mathrm{r}^{2}$ value was only 0.032, indicating that the significance of prematurity to UCVA might not be very high. SES factors, such as the employment status of parents and family income, which were significantly associated with UCVA in MEPEDS and SPEDS, showed no associations with UCVA in our study. In addition, maternal smoking during pregnancy, which was related with slightly better UCVA in SPEDS, had no relationship with UCVA in our study.

As the penalty for missing one amblyopia case is a lifetime disability and treatment exists, the UCVA referral cut-off for amblyopia should target high sensitivity, while the specificity should be high enough not to put immense load on ophthalmologists. ${ }^{24}{ }^{25}$ The sensitivity of detecting amblyopia by using the UCVA referral cut-off in our study (UCVA $\leq 20 / 40$ at age 36 to $<48$ months) was as high as $86 \%$, and at the same time, the specificity was high enough $(74.2 \%)$. On the other hand, this cut-off only identified a total of $39.5 \%$ refractive errors, even though the overall specificity was high $(84.5 \%)$. The sensitivity in detecting myopia was not good in our study $(35.1 \%)$, lower than hyperopia (51.2), astigmatism $(48.6 \%)$ and anisometropia $(44.1 \%)$. In SKES, even though a high sensitivity $(83.3 \%)$ in detecting myopia was observed in all 36-month-old to 72-month-old 
preschoolers, it is not available to calculate the sensitivity in detecting myopia in 36-month-old children because of no presence of myopia in this age group. And for all refractive errors in 36-month-old children in SKES, the overall sensitivity was only $8.6 \%$. Therefore, only using the UCVA cut-off as referral criterion might be difficult to detect refractive errors in 36-month-old children, and additional refraction examination might be necessary for vision screening.

The normative distribution of UCVA IOD in our study was consistent with that in SKES, with most children achieving equal vision in both eyes. No effect of gender or testing order on IOD was observed in our study, which was consistent with MEPEDS. However, we found that absolute UCVA IOD decreased with increasing age of month in our sample, which was not consistent with the findings in 30-month-old to 72-month-old children in MEPEDS. The reason for this difference might be that, in MEPEDS, equal vision in both eyes in older children concealed the effect of age on UCVA IOD in younger children.

When using UCVA IOD of two or more lines as the criterion, approximately $4 \%$ in the general population would be referred in both of SKES and our study. The sensitivity of using this criterion alone for detecting amblyopia, including unilateral amblyopia, was very low in our study, similar to the results in SKES.

To our knowledge, the present study is the first to provide population-based HOTV-specific normative UCVA data in Chinese preschoolers aged 36 to $<48$ months without significant refractive error or other ophthalmic abnormalities. However, there are several limitations in the present study. Because young children are very afraid of using eye drops, which makes cycloplegia difficult and unacceptable for parents, only children with suspected abnormalities underwent cycloplegic refraction in our study. Even though not all children had refraction results under cycloplegia, we adopted stringent criteria to identify children who needed cycloplegic refraction, which could effectively detect sight-affecting refractive errors. However, Fotedar $e t a l^{20}$ and Leone $e t a l^{21}$ found that hyperopia and some astigmatism might not be as detectable without cycloplegia, which might impact the findings of our reference population at some degree. $11.1 \%$ children were not testable when doing the HOTV test and were excluded from the analysis, which may influence the normative UCVA distribution. However, the testability in our study was at an average level of published reports, ${ }^{6}{ }^{10}$ which tested VA using the HOTV single surrounded letters, not linear, and difficult to increase further in young children.

In conclusion, our study suggests that Chinese preschoolers aged 36 to $<48$ months with UCVA no better than 20/40 measured by linear HOTV chart should be referred for further ophthalmic examinations. Most amblyopia cases can be identified with this age-specific and chart-specific UCVA cut-off.
Acknowledgements We thank the children, the corresponding parents or legal guardians and all the members of the Maternal and Child Healthcare Hospital of Yuhuatai District, Nanjing, China, for helpful advice and support.

Contributors Conceived and designed the experiments: $\mathrm{HL}, \mathrm{HZ}, \mathrm{DH}$ and QS. Performed the experiments: $\mathrm{HZ}, \mathrm{DH}, \mathrm{QS}, \mathrm{HD}, \mathrm{JB}, \mathrm{JC}, \mathrm{YW}, \mathrm{XZ}$, JW and XL. Analysed the data: $\mathrm{HZ}, \mathrm{DH}, \mathrm{QS}$ and $\mathrm{XC}$. Contributed reagents/materials/analysis tools: $\mathrm{HL}, \mathrm{HZ}$, $\mathrm{DH}, \mathrm{HD}, \mathrm{JB}, \mathrm{JC}$, and XC. Wrote the paper: HL, HZ, DH, QS, XC, HD, JB, JC, YW, XZ, JW and $\mathrm{XL}$.

Funding This study is supported by Scientific Research Projects of Jiangsu Provincial Commission of Health and Family Planning (Grant no H201507); Natural Science Foundation of Jiangsu Province (Grant nos BK20141027 and BK20161595); National Natural Science Foundation of China (Grant nos 81400435 and 81673198).

Competing interests None declared.

Patient consent Guardian consent obtained

Ethics approval The Institutional Review Board of Jiangsu Province Hospital.

Provenance and peer review Not commissioned; externally peer reviewed.

Data sharing statement № additional data are available.

Open Access This is an Open Access article distributed in accordance with the Creative Commons Attribution Non Commercial (CC BY-NC 4.0) license, which permits others to distribute, remix, adapt, build upon this work non-commercially, and license their derivative works on different terms, provided the original work is properly cited and the use is non-commercial. See: http://creativecommons.org/ licenses/by-nc/4.0/

(c) Article author(s) (or their employer(s) unless otherwise stated in the text of the article) 2017. All rights reserved. No commercial use is permitted unless otherwise expressly granted.

\section{REFERENCES}

1 Hartmann EE, Dobson V, Hainline L, et al. Preschool vision screening: summary of a Task Force report. behalf of the maternal and Child Health Bureau and the National Eye Institute Task Force on Vision Screening in the Preschool Child. Pediatrics 2000;106:1105-16.

2 American Academy of Pediatrics, Committee on Practice and Ambulatory Medicine and Section on Ophthalmology. Eye examination in infants, children, and young adults by pediatricians. Pediatrics 2003;111:902-7.

3 Lai YH, Hsu HT, Wang HZ, et al. The visual status of children ages 3 to 6 years in the vision screening program in Taiwan. $J$ Aapos 2009;13:58-62.

4 Hård AL, Sjödell L, Borres MP, et al. Preschool vision screening in a swedish city region: results after alteration of criteria for referral to eye clinics. Acta Ophthalmol Scand 2002;80:608-11.

5. Donahue SP, Arthur B, Neely DE, et al. Guidelines for automated preschool vision screening: a 10-year, evidence-based update. $J$ Aapos 2013;17:4-8.

6 Pan Y, Tarczy-Hornoch K, Cotter SA, et al. Visual acuity norms in preschool children: the Multi-Ethnic Pediatric Eye Disease Study. Optom Vis Sci 2009;86:607-12.

7. Leone JF, Mitchell P, Kifley A, et al. Normative visual acuity in infants and preschool-aged children in Sydney. Acta Ophthalmol 2014;92:e521-e529.

8 Friedman DS, Repka MX, Katz J, et al. Prevalence of decreased visual acuity among preschool-aged children in an american urban population: the Baltimore Pediatric Eye Disease Study, methods, and results. Ophthalmology 2008;115:1786-95.

9 Robaei D, Rose K, Ojaimi E, et al. Visual acuity and the causes of visual loss in a population-based sample of 6-year-old australian children. Ophthalmology 2005;112:1275-82.

10 Guo X, Fu M, Lu J, et al. Normative distribution of visual acuity in 3to 6-year-old chinese preschoolers: the Shenzhen kindergarten eye study. Invest Ophthalmol Vis Sci 2015;56:1985-92.

11. Cryotherapy for Retinopathy of Prematurity Cooperative Group. Multicenter trial of cryotherapy for retinopathy of prematurity. Snellen visual acuity and structural outcome at $51 / 2$ years after randomization. Arch Ophthalmol 1996;114:417-24.

12 Hartmann EE, Bradford GE, Chaplin PK, et al. PUPVS Panel for the American Academy of Pediatrics. Project Universal Preschool Vision Screening: a demonstration project. Pediatrics 2006;117:e226-e237.

13 Huang D, Chen X, Gong Q, et al. Ocular biometric parameters among 3-year-old Chinese children: testability, distribution and association with anthropometric parameters. Sci Rep 2016;6:29577. 
14. Holmes JM, Beck RW, Repka MX, et al. The amblyopia treatment study visual acuity testing protocol. Arch Ophthalmol 2001;119:1345-53.

15 American academy of Ophthalmology Pediatric Ophthalmology/ Strabismus Panel. Preferred practice pattern guidelines. Amblyopia. San Francisco, CA: American Academy of Ophthalmology, 2012. www.aao.org/ppp

16. Society CO. Guidelines for amblyopia diagnosis. Chin J Ophthalmol 2011; $47: 768$

17 He M, Zeng J, Liu Y, et al. Refractive error and visual impairment in urban children in southern china. Invest Ophthalmol Vis Sci 2004;45:793-9.

18 Drover JR, Felius J, Cheng CS, et al. Normative pediatric visual acuity using single surrounded HOTV optotypes on the electronic visual acuity tester following the Amblyopia treatment study protocol. $J$ Aapos 2008;12:145-9.

19. Rice ML, Leske DA, Holmes JM. Comparison of the amblyopia treatment study HOTV and electronic-early treatment of diabetic retinopathy study visual acuity protocols in children aged 5 to 12 years. Am J Ophthalmol 2004;137:278-82.
20. Fotedar R, Rochtchina E, Morgan I, et al. Necessity of cycloplegia for assessing refractive error in 12-year-old children: a population-based study. Am J Ophthalmol 2007;144:307-9.

21. Leone JF, Mitchell P, Morgan IG, et al. Use of visual acuity to screen for significant refractive errors in adolescents: is it reliable? Arch Ophthalmol 2010;128:894-9.

22 Leone JF, Gole GA, Mitchell P, et al. Visual acuity testability and comparability in australian preschool children: the Sydney Paediatric Eye Disease Study. Eye 2012;26:925-32.

23 Dobson V, Clifford-Donaldson CE, Green TK, et al. Normative monocular visual acuity for early treatment diabetic retinopathy study charts in emmetropic children 5 to 12 years of age. Ophthalmology 2009:116:1397-401.

24. Kvarnström G, Jakobsson P, Lennerstrand G. Screening for visual and ocular disorders in children, evaluation of the system in Sweden. Acta Paediatr 1998;87:1173-9.

25. Bušić $M$, Bjeloš $M$, Petrovečki $M$, et al. Zagreb Amblyopia Preschool Screening Study: near and distance visual acuity testing increase the diagnostic accuracy of screening for amblyopia. Croat Med $J$ 2016;57:29-41. 